\title{
Prolonged Suppressive Effects of Periodontitis on Salivary TFF3 Production
}

\author{
Doosadee Hormdee ${ }^{1,2}$ Saengsome Prajaneh ${ }^{1} \quad$ Amonrujee Kampichai ${ }^{3} \quad$ Ranuch Tak $^{4}$ \\ Ponlatham Chaiyarit ${ }^{2,5}$
}

${ }^{1}$ Division of Periodontology, Department of Oral Biomedical Sciences, Faculty of Dentistry, Khon Kaen University, Khon Kaen, Thailand

${ }^{2}$ Research Group of Chronic Inflammatory Oral Diseases and Systemic Diseases Associated with Oral Health, Khon Kaen University, Thailand

${ }^{3}$ Division of Dentistry, Fang Hospital, Chiang Mai, Thailand

${ }^{4}$ Faculty of Dentistry, International University, Phnom Penh, Cambodia

${ }^{5}$ Division of Oral Diagnosis, Department of Oral Biomedical Sciences, Faculty of Dentistry, Khon Kaen University, Khon Kaen, Thailand

Eur J Dent 2019;13:193-198
Address for correspondence Doosadee Hormdee, DDS, PhD, Department of Oral Biomedical Sciences, Division of Periodontology, Faculty of Dentistry, Khon Kaen University, 123 Mitaparp Road, T.Naimuang, U.Muang, Khon Kaen, 40002, Thailand (e-mail: nootdoosadee@hotmail.com).

\section{Abstract}

Keywords

- periodontal diseases

- saliva

- salivary glands

- trefoil factor
Objective As a follow-up to our previous study that demonstrated decreased salivary trefoil factor family 3 (TFF3) peptide levels in chronic periodontitis patients, this current study aimed to observe the effects of nonsurgical periodontal treatment on salivary TFF3 peptides in patients with periodontal diseases.

Materials and Methods Eighty-seven volunteers that comprised of 30 individuals with healthy periodontium, 31 with gingivitis, and 26 with chronic periodontitis were considered for the study. Prior to periodontal treatment, a general periodontal examination was performed along with collection of saliva samples from each volunteer. Nonsurgical periodontal treatments were provided to patients with gingivitis and periodontitis. Two weeks post-treatment, saliva samples were recollected, and the periodontal status was re-evaluated. Salivary TFF3 concentrations were measured by enzyme-linked immunosorbent assay.

Statistical Analysis Mann-Whitney U test was used when the investigated data were not normally distributed. Chi-squared test was used when dealing with categorical data. Kruskal-Wallis test with post-hoc corrections was used to compare data among the three investigated groups. Two-tailed $p<0.05$ was considered as statistically significant.

Results Prior to the periodontal treatment, salivary TFF3 concentrations in patients with gingivitis and periodontitis were significantly lower than those with healthy periodontium. Two weeks post-treatment, increased levels of salivary TFF3 were observed in patients with gingivitis, whereas the concentrations decreased in patients with chronic periodontitis.

Conclusion This study demonstrated the effects of periodontal disease on the production of salivary TFF3 peptides. Interestingly, nonsurgical periodontal treatment also affected the recovery of salivary TFF3 peptides but varied in their outcomes between gingivitis and periodontitis patients.
DOI https://doi.org/

10.1055/s-0039-1693949

ISSN 1305-7456.
License terms

()(1) $\ominus \circledast$ 


\section{Introduction}

Trefoil factor family (TFF) peptides are composed of TFF1, TFF2, and TFF3. Members of TFF peptides share a common molecular structure known as a trefoil domain. ${ }^{1}$ It was reported that an interaction between a free cysteine residue in the C-terminal of this TFF peptide and other proteins would alter biological properties and activities of the TFF molecules. ${ }^{2}$ The distribution and localization of TFF peptides vary according to organs, tissues, and body fluids. ${ }^{3,4}$ These peptides have several biological functions including cell migration ${ }^{5}$ and wound healing. ${ }^{6}$ Results from animal studies have shown that TFF3 modulated inflammation by interfering the production and secretion of inflammatory cytokines, such as interleukin-1beta (IL-1 $\beta$, IL-6, and IL-8., Recombinant human TFF3 peptides also inhibit the production of tolllike receptor 4, nuclear factor kappa B (NF-KB), and tumor necrosis factor alpha (TNF- $\alpha$ in epithelia of colitis mice. ${ }^{9}$ In contrast, it was demonstrated that NF- $\kappa B$ signaling pathway was related with the downregulation of TFF expression. ${ }^{10}$ The TFF peptides have been intensively investigated in the gastrointestinal tract and are considered to exert their functions in maintenance and protection of mucosal tissues. ${ }^{11}$ However, information on TFF peptides in the oral cavity is limited.

TFF peptides are identified in different oral tissues including salivary glands, ${ }^{12}$ oral mucosa, ${ }^{13}$ gingiva,${ }^{14}$ and saliva. ${ }^{4}$ They are mainly produced from the salivary glands ${ }^{15}$ with some addition from the parotid duct and oral mucosal epithelia. ${ }^{16}$ Among salivary TFF peptides, TFF3 is the most prominent, followed by TFF1 and TFF2. ${ }^{4}$ The previous report explained that TFF3 was a modifying factor involved in oral keratinocytes signaling pathways, such as cell survival, cell proliferation, and cell migration. ${ }^{17}$ Therefore, presence of TFF peptides in saliva may be crucial for the protection of oral mucosal against tissue damage. Previously, our cross-sectional study demonstrated reduced salivary TFF3 peptides in chronic periodontitis (CP) subjects, and the levels of TFF3 negatively correlated with the severity of periodontitis. ${ }^{14}$ Additionally, our in vitro study has revealed that TFF peptides could be digested by major proteolytic enzymes produced by periopathogenic bacteria. ${ }^{18}$ Taking these findings into account, periodontal inflammation mediated by periodontopathic bacteria may be a downregulating factor in the production of salivary TFF3 peptides in patients with CP. We hypothesized that reduction of periodontal inflammation by nonsurgical periodontal treatment would elevate the production of salivary TFF3 peptides. The present study was aimed to verify our hypothesis by examining salivary TFF3 peptide levels in gingivitis and periodontitis subjects prior to and following completion of nonsurgical periodontal treatment.

\section{Materials and Methods}

\section{Study Population and Clinical Examination}

This prospective study was performed at the Dental Hospital, Khon Kaen University, Thailand during 2014 to 2016. All procedures were approved by Khon Kaen University Ethics committee (HE551372). Eighty-seven systemically healthy volunteers including $26 \mathrm{CP}$ patients, 31 dental plaque induced gingival diseased (GD) patients, and 30 clinically periodontally healthy (PH) individuals participated in this study. Written informed consents were obtained from all participants. All subjects were nonsmokers who had at least 15 remaining teeth. A general periodontal examination periodontal parameters including bleeding on probing (BOP), probing depth (PD), and clinical attachment level (CAL) on six sites of each remaining tooth, except third molar, were measured and recorded by one periodontist. Diagnosis of periodontal diseases was based on the 1999 International Workshop for classification of Periodontal Diseases and Conditions. ${ }^{19}$ Two weeks following completion of the conventional periodontal treatment, a second full-mouth general periodontal examination was performed and the parameters were recorded.

\section{Saliva Collection}

Saliva collection was performed as previously described. ${ }^{14}$ Each volunteer was asked to refrain from eating or drinking for at least 1 hour prior to saliva collection. After rinsing the mouth thoroughly with water, unstimulated whole saliva sample (3-5 mL) was obtained in a $50 \mathrm{~mL}$ centrifuge tube. The first saliva collection was done in the morning during 9 to $11 \mathrm{am}$, followed by the first periodontal examination for each volunteer. Two weeks post-treatment, second saliva collection and periodontal examination were performed with the GD and $\mathrm{CP}$ groups. Saliva samples were immediately placed in an ice container, followed by centrifugation at $10,000 \times \mathrm{g}$ at $4^{\circ} \mathrm{C}$ for 10 minutes. Saliva samples were stored at $-80^{\circ} \mathrm{C}$ until further measurements. Total protein concentrations from each saliva sample were determined employing a commercially available protein assay kit (Qubit Protein Assay kit; Thermo Fisher Scientific, Invitogen, United States) to test whether any differences in salivary TFF3 concentrations could be referred to the differences in the total salivary protein concentrations.

\section{Measurement of Salivary TFF3 Concentrations}

For the quantification of salivary TFF3 concentrations, we used our generated monoclonal antibody (mAb) clones 286 and 116 for a modified sandwich enzyme-linked immunosorbent assay (ELISA) technique. ${ }^{20}$ To develop the sensitivity, fluorescein isothiocyanate (FITC) and anti-FITC identification methods were used in this sandwich ELISA technique. An antihuman TFF3 mAb clone 286 was used as the plate-coating antibody to capture the peptides in saliva samples. The antihuman TFF3 mAb clone 116 labeled with FITC was applied to detect the captured TFF3 peptides. Horseradish peroxidase (HRP)-conjugated sheep anti-FITC antibodies along with TMB substrate were used as colored markers. The color intensity was measured using a microplate reader at $450 \mathrm{~nm}$. A standard curve revealing salivary TFF3 between 0.5 and $32 \mathrm{ng} / \mathrm{mL}$ was observed. All details of salivary TFF3 concentrations were normalized by total salivary protein concentrations and reported as TFF3 concentrations (ng)/salivary protein concentration (mg).

\section{Nonsurgical Periodontal Treatments}

Nonsurgical mechanical treatments were provided for gingivitis and periodontitis patients in the periodontal clinic, 
Faculty of Dentistry, Khon Kaen University. The treatment included plaque control by providing oral hygiene instructions and mechanical debridement by full-mouth scaling and root planing. ${ }^{21-23}$ Periodontal treatments were performed in single or multiple visits, utilizing manual curettes (Hu-Friedy, Chicago, Illinois, United States) and ultrasonic instruments (P5 Newtron, Acteon, France). Two weeks following completion of nonsurgical periodontal treatment, the periodontal health was re-examined by the same periodontist.

\section{Statistical Analysis}

IBM SPSS software version 19.0 was used for the statistical analyses. Student's $t$-test was used to evaluate normally distributed data between the two groups, whereas MannWhitney $U$ test was used when the investigated data were not normally distributed. Chi-squared test was used when dealing with categorical data. Kruskal-Wallis test with posthoc corrections was used to compare data among the three investigated groups. Spearman's rank correlation was used to evaluate correlations between salivary TFF3 peptides levels and periodontal parameters. Two-tailed $p<0.05$ was considered as statistically significant.

\section{Results}

\section{Demographic and Clinical Characteristics of the Study Population}

Demographic and clinical characteristics of three investigated groups were as shown in $\mathbf{- T a b l e s} \mathbf{1}$ and $\mathbf{2}$. The mean age of PH individuals and GD patients was similar. In contrast, the mean age of $\mathrm{CP}$ patients was significantly higher than those in the PH and GD groups. Females were predominant in all the three groups. Prior to the nonsurgical periodontal

Table 1 Demographic data of the study population

\begin{tabular}{|l|l|l|l|l|}
\hline \multirow{2}{*}{} & \multicolumn{4}{|c|}{ Investigated groups } \\
\cline { 2 - 5 } & Healthy & Gingivitis & Periodontitis & $p$-Value \\
\hline Number & 30 & 31 & 26 & \\
\hline Age in years (mean \pm SD) & $32.19 \pm 1.61$ & $32.13 \pm 1.81$ & $44.04 \pm 2.74$ & $0.001^{\text {a }}$ \\
\hline Male & 5 & 5 & 9 & \\
\hline Female & 25 & 26 & 14 & $0.085^{\mathrm{b}}$ \\
\hline
\end{tabular}

Abbreviations: ANOVA, analysis of variance; SD, standard deviation.

${ }^{a}$ Kruskal-Wallis one-way ANOVA.

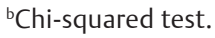

Table 2 Clinical parameters and salivary characteristic data of the investigated groups prior to and 2 weeks following nonsurgical periodontal treatment

\begin{tabular}{|c|c|c|c|c|c|}
\hline \multicolumn{2}{|l|}{ Clinical parameters } & \multicolumn{4}{|c|}{ Investigated groups } \\
\hline & & \multirow{2}{*}{$\begin{array}{l}\text { Healthy }(n=30) \\
1.89 \pm 0.19\end{array}$} & \multirow{2}{*}{$\begin{array}{l}\text { Gingivitis }(n=31) \\
1.94 \pm 0.16\end{array}$} & \multirow{2}{*}{$\begin{array}{l}\text { Periodontitis }(n=26) \\
2.64 \pm 0.59\end{array}$} & \multirow{2}{*}{$\begin{array}{l}p \text {-Values } \\
<0.001^{\text {a }}\end{array}$} \\
\hline PD (mm; mean \pm SD) & Before treatment & & & & \\
\hline & After treatment & - & $1.86 \pm 0.14$ & $1.98 \pm 0.25$ & $<0.001^{c}$ \\
\hline \multirow[t]{2}{*}{ CAL (mm; mean \pm SD) } & Before treatment & $0.52 \pm 1.77$ & $0.55 \pm 0.12$ & $2.35 \pm 1.07$ & $<0.001^{\mathrm{a}}$ \\
\hline & After treatment & - & $0.54 \pm 0.14$ & $2.09 \pm 0.92$ & $0.358^{d}$ \\
\hline \multirow[t]{2}{*}{$\mathrm{BOP}($ mean $\pm \mathrm{SD})$} & Before treatment & $15.42 \pm 3.57$ & $38.28 \pm 9.98$ & $51.7 \pm 15.29$ & $<0.001^{\mathrm{a}}$ \\
\hline & After treatment & - & $23.02 \pm 6.93$ & $30.47 \pm 12.65$ & $0.015^{d}$ \\
\hline \multicolumn{6}{|l|}{ Salivary characteristics } \\
\hline \multirow{2}{*}{$\begin{array}{l}\text { Total salivary protein } \\
\text { concentrations (mg/ } \\
\text { mL: mean } \pm \text { SD) }\end{array}$} & Before treatment & $0.59 \pm 0.28$ & $0.57 \pm 0.31$ & $0.73 \pm 0.76$ & $0.775^{\mathrm{a}}$ \\
\hline & After treatment & - & $0.49 \pm 0.16$ & $0.75 \pm 0.29$ & $<0.001^{c}$ \\
\hline \multirow[t]{2}{*}{$\mathrm{pH}$} & Before treatment & $6.99 \pm 0.24$ & $6.98 \pm 0.29$ & $6.79 \pm 0.4$ & $0.083^{\mathrm{a}}$ \\
\hline & After treatment & - & $6.89 \pm 0.3$ & $6.76 \pm 0.36$ & $0.153^{d}$ \\
\hline \multirow{2}{*}{$\begin{array}{l}\text { Normalized salivary } \\
\text { TFF3 concentrations } \\
\text { (ng/mg protein) }\end{array}$} & Before treatment & $228.46 \pm 149.0$ & $93.05 \pm 83.33$ & $156.73 \pm 147.23$ & $<0.001^{\mathrm{a}}$ \\
\hline & After treatment & - & $252.73+171.17$ & $40.74+41.77$ & $<0.001^{d}$ \\
\hline
\end{tabular}

Abbreviations: ANOVA, analysis of variance; BOP, bleeding on probing; PD, probing depth; CAL, clinical attachment level; TFF3, trefoil factor family 3; SD, standard deviation.

aruskal-Wallis one-way ANOVA.

${ }^{\mathrm{b} C h i-s q u a r e d ~ t e s t . ~}$

'Mann-Whitney $U$ test.

¿Student's t-test. 
treatment, the total salivary protein concentrations were not significantly different among the three groups. Following treatment, salivary protein concentrations in the GD group were significantly different than those in the CP group $(p<$ $0.001)$. In regard to the periodontal parameters prior to the treatments, the mean BOP value in the PH group was significantly lower than the GD and CP groups $(p<0.001)$. PD and CAL measures from the $\mathrm{CP}$ group were significantly greater than those from PH and GD groups $(p<0.001)$. Improvement in periodontal health status at 2 weeks following the treatments was observed in GD and CP groups.
Levels of Salivary TFF3 Peptides in GD and CP Groups before and after Nonsurgical Periodontal Treatments

The median, mean, upper, and lower quartiles values of normalized salivary TFF3 concentrations were as shown in

-Fig. 1. The trend of alterative salivary TFF3 in the GD and $\mathrm{CP}$ groups following nonsurgical periodontal treatment was as shown in - Fig. 2. Prior to the periodontal treatment, mean normalized salivary TFF3 concentrations in PH, GD, and CP groups were 228.46, 93.05, and $156.73 \mathrm{ng} / \mathrm{mg}$, respectively. Normalized salivary TFF3 concentrations in the PH group were significantly higher than the GD and CP groups

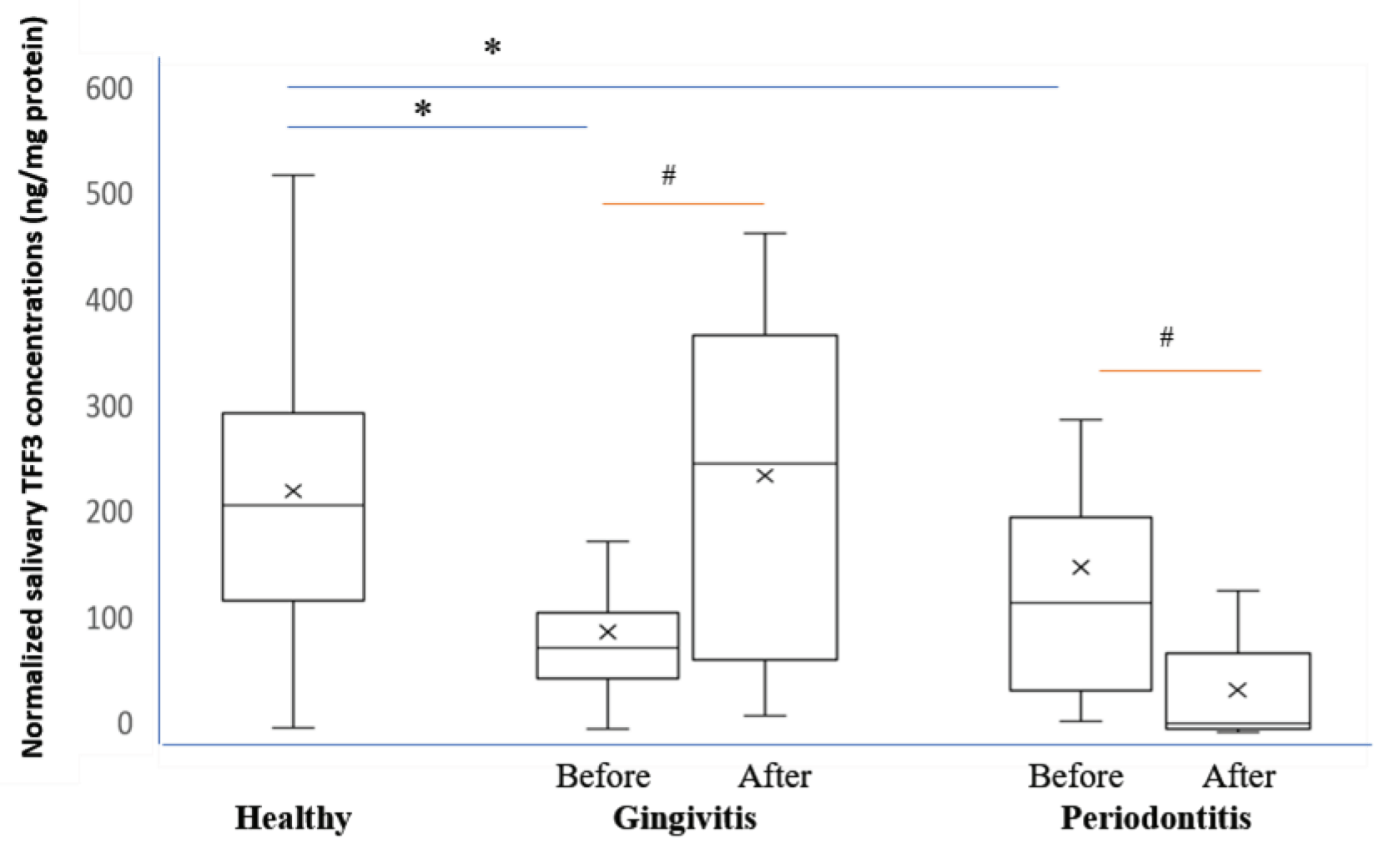

Fig. 1 Normalized salivary Trefoil factor family 3 (TFF3) concentrations (ng/mg) among the three investigated groups including clinically periodontal healthy (healthy, $n=30$ ); gingival diseased (gingivitis, $n=31$ ); and chronic periodontitis (periodontitis, $n=26$ ) individuals prior to and 2 weeks following nonsurgical periodontal treatment. Middle line indicates median, cross indicates mean, box indicates the upper and lower quartiles, and whiskers indicate minimum and maximum values. The asterisk represents the $p$-value $<0.05$ (Mann-Whitney U test). The sharp represents the $p$-value $<0.001$ (Wilcoxon signed-rank test).
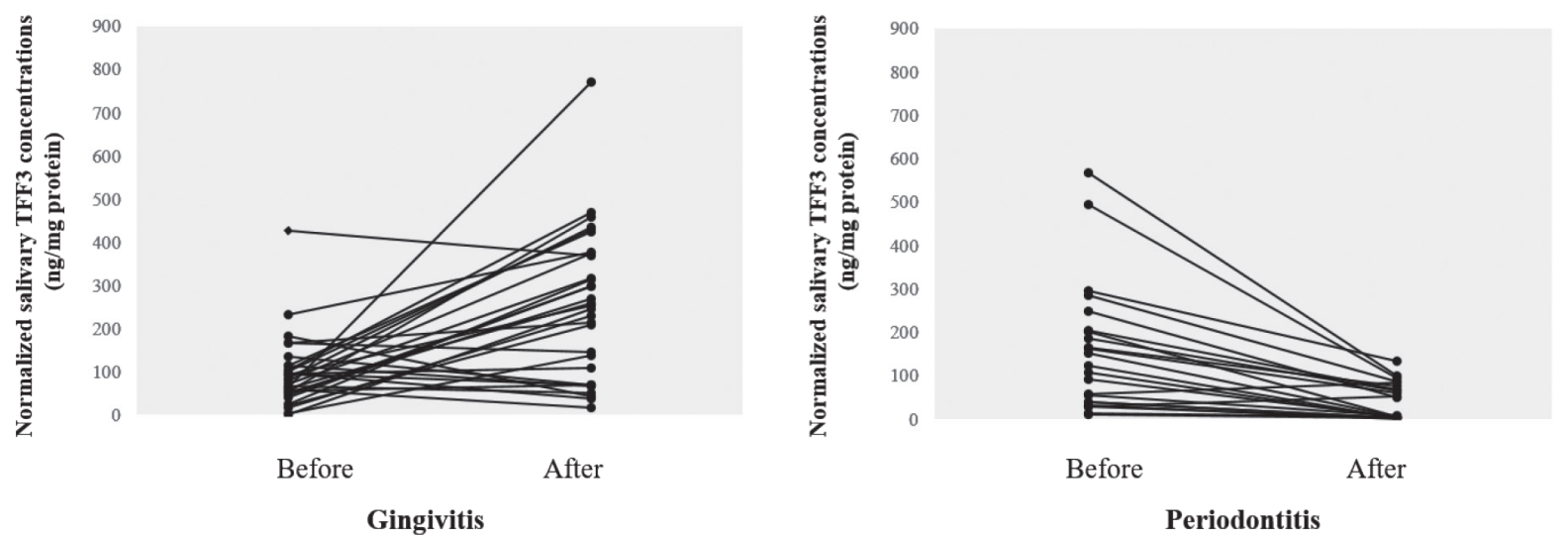

Fig. 2 A trend of alterative salivary trefoil factor family 3 (TFF3) in the gingival disease (gingivitis, $n=31$ ) and chronic periodontitis (periodontitis, $n=26$ ) patients prior to and 2 weeks following nonsurgical periodontal treatment. 
$(p<0.05)$. Two weeks following completion of periodontal treatment in the GD group, there was a significant increase in the salivary TFF3 $(252.73 \mathrm{ng} / \mathrm{mg})(p<0.001)$. The level of salivary TFF3 peptides in the GD group after receiving the treatment was comparable with those in the PH group. On the other hand, salivary TFF3 peptides in the CP group after receiving the treatment significantly decreased as compared with those before the treatment $(p<0.001)$.

\section{Correlations between Salivary TFF3 Concentrations and Periodontal Parameters}

Spearman's rank correlation analysis was performed on all the subjects with periodontal diseases. Prior to the periodontal treatment, significantly negative correlations were observed between salivary TFF3 levels and BOP (correlation coefficient $=-0.379, p=0.005$ ). On the other hand, no correlation was observed between salivary TFF3 levels and other periodontal parameters. Likewise, following periodontal treatment, no correlation was demonstrated between salivary TFF3 levels and periodontal parameters.

\section{Discussion}

According to our observations, the levels of salivary TFF3 peptides in gingivitis and periodontitis patients prior to the periodontal treatment were lower than those in PH subjects. These results were in agreement with our previous study ${ }^{14}$ and suggested that decreased levels of salivary TFF3 peptides could be due to local and systemic effects of periodontal diseases. Regarding the local effects, our previous in vitro study demonstrated proteolytic activity of gingipains, one of the major proteolytic enzymes produced by Porphyromonas gingivalis, on digesting TFF peptides. ${ }^{18}$ These findings imply that periodontopathic enzymes may locally reduce TFF3 peptides in saliva. As TFF3 peptides are mainly produced by salivary glands, decreased levels of salivary TFF3 peptides may reflect the systemic impact of periodontal diseases on the functions of the salivary gland. Several investigations have demonstrated the association between periodontal diseases and biological functions of salivary glands. ${ }^{24,25}$ One study demonstrated hyposalivation in experimental periodontitis model rats. ${ }^{24}$ Similarly, alterations of inflammatory microRNA in salivary glands of rats infected with periodontal bacteria have also been observed..$^{25}$ Our findings provide new implication that periodontal diseases may be a downregulating factor in the production of TFF3 from the salivary glands. Regarding the pathological effects of periodontal diseases on salivary gland dysfunctions, further studies to validate correlations between periodontal diseases and protein synthesis from salivary glands would be of importance.

The effects of nonsurgical periodontal treatments on salivary compositions were investigated in patients with periodontitis, and outcomes were varied..$^{26-29}$ Some studies reported changes in salivary components, ${ }^{26,27,30}$ whereas others demonstrated no significant impact of nonsurgical periodontal treatment on salivary components. ${ }^{25,26}$ Nonsurgical periodontal treatments have been observed to improve the levels of proinflammatory cytokines, including TNF- $\alpha$ and endothelial growth factor $A$, in serum and gingival crevicular fluid. ${ }^{31}$ In our results, nonsurgical periodontal treatment increased levels of salivary TFF3 peptides in gingivitis patients to the same levels as in $\mathrm{PH}$ individuals. Taking these findings into account, it could be possible that gingival inflammation could be a temporary downregulator of salivary TFF3 production, and the reduction in proinflammatory cytokines related to gingivitis by nonsurgical periodontal treatments would help to regain salivary TFF3 production. In contrast, the periodontal therapy had no effect on the recovery of salivary TFF3 peptides in periodontitis patients. These results are indicative of the suppressive effects of periodontitis on the functioning of salivary glands and reflect differential effects of nonsurgical periodontal treatment on the production of salivary TFF3 peptides in periodontitis patients as compared with those in gingivitis patients. However, it should be noted that the follow-up period in this study was 2 weeks following periodontal treatment. It is possible that at this time point the ongoing inflammatory response due to $\mathrm{CP}$ may still remain in effect and could interrupt the recovery of salivary glands' functions. Thus, an extended follow-up study should be conducted to re-examine the levels of salivary TFF3 peptides.

Previous studies demonstrated that TFF3 peptides had a protective effect on mucosal tissues by the downregulation of proinflammatory cytokines. ${ }^{7-9}$ It was reported that recombinant human TFF3 administered as a topical oral spray could reduce oral mucositis in colorectal cancer patients who received fluorouracil-based chemotherapy. ${ }^{32}$ Thus, the presence of salivary TFF3 peptides may be an important protective factor against oral mucosal tissue damage. However, it remains unclear whether salivary TFF3 plays any role in periodontal diseases. Our previous observations ${ }^{14}$ combined with the present results demonstrated inverse correlations between levels of salivary TFF3 peptides and periodontal parameters in patients with periodontal diseases. It would be of interest to further investigate whether administration of topical TFF3 peptides could help to reduce periodontal inflammation.

In conclusion, the present study demonstrated the impact of periodontal diseases on the production of salivary TFF3 peptides, and confirmed our previous findings of decreased levels of salivary TFF3 peptides in patients with periodontal diseases. Nonsurgical periodontal treatments had varied results on the recovery of salivary TFF3 peptides between gingivitis and periodontitis patients. The treatment elevated salivary TFF3 peptides in gingivitis patients but the treatment had no effect on the recovery of salivary TFF3 peptides in periodontitis patients. Our findings provide a new implication that periodontal diseases may be a downregulator of salivary glands in the context of TFF3 production.

\section{Funding}

This work was supported by the TRF Mid-Career Research Scholar (RSA5680010) and Khon Kaen University (KKU 600301). 


\section{Conflict of Interest}

None declared.

\section{References}

1 Hoffmann W, TFF peptides. In: Kastin AJ, ed. Handbook of Biologically Active Peptides. Elsevier; 2013:1338-1345

2 Muskett FW, May FE, Westley BR, Feeney J. Solution structure of the disulfide-linked dimer of human intestinal trefoil factor (TFF3): the intermolecular orientation and interactions are markedly different from those of other dimeric trefoil proteins. Biochemistry 2003;42(51):15139-15147

3 Madsen J, Nielsen O, Tornøe I, Thim L, Holmskov U. Tissue localization of human trefoil factors 1, 2, and 3. J Histochem Cytochem 2007;55(5):505-513

4 Samson $\mathrm{MH}$, Chaiyarit P, Nortvig H, Vestergaard EM, Ernst E, Nexo E. Trefoil factor family peptides in human saliva and cyclical cervical mucus. Method evaluation and results on healthy individuals. Clin Chem Lab Med 2011;49(5):861-868

5 Marchbank T, Playford RJ. Trefoil factor family peptides enhance cell migration by increasing cellular osmotic permeability and aquaporin 3 levels. FASEB J 2018;32(2):1017-1024

6 Schulze U, Hampel U, Sel S, et al. Trefoil factor family peptide 3 (TFF3) is upregulated under experimental conditions similar to dry eye disease and supports corneal wound healing effects in vitro. Invest Ophthalmol Vis Sci 2014;55(5):3037-3042

7 Shi L, Zhou PH, Xi JL, Yu HG, Zhang BH. Recombinant human trefoil factor 3 ameliorates bowel injury: its anti-inflammatory effect on experimental necrotizing enterocolitis. Int J Pept 2014;2014:634135

8 Barrera GJ, Sanchez G, Gonzalez JE. Trefoil factor 3 isolated from human breast milk downregulates cytokines (IL8 and IL6) and promotes human beta defensin (hBD2 and hBD4) expression in intestinal epithelial cells HT-29. Bosn J Basic Med Sci 2012;12(4):256-264

9 Teng X, Xu LF, Zhou P, Sun HW, Sun M. Effects of trefoil peptide 3 on expression of TNF-. $\alpha$. , TLR4, and NF-kappaB in trinitrobenzene sulphonic acid induced colitis mice. Inflammation 2009;32(2):120-129

10 Dossinger V, Kayademir T, Blin N, Gött P. Down-regulation of TFF expression in gastrointestinal cell lines by cytokines and nuclear factors. Cell Physiol Biochem 2002;12(4):197-206

11 Aihara E, Engevik KA, Montrose MH. Trefoil factor peptides and gastrointestinal function. Annu Rev Physiol 2017;79:357-380

12 Chaiyarit P, Klanrit P, Photipakdee P, Subarnbhesaj A, Giraud AS. Increased immunoexpression of trefoil factors in salivary gland tumors. Clin Oral Investig 2014;18(4):1305-1312

13 Chaiyarit P, Utrawichian A, Leelayuwat C, et al. Investigation of trefoil factor expression in saliva and oral mucosal tissues of patients with oral squamous cell carcinoma. Clin Oral Investig 2012;16(6):1549-1556

14 Chaiyarit P, Chayasadom A, Wara-Aswapati N, et al. Trefoil factors in saliva and gingival tissues of patients with chronic periodontitis. J Periodontol 2012;83(9):1129-1138

15 Devine DA, High AS, Owen PJ, Poulsom R, Bonass WA. Trefoil factor expression in normal and diseased human salivary glands. Hum Pathol 2000;31(4):509-515

16 Kutta H, May J, Jaehne M, Münscher A, Paulsen FP. Antimicrobial defence mechanisms of the human parotid duct. J Anat 2006;208(5):609-619
17 Storesund T, Schreurs O, Messelt EB, Kolltveit KM, Schenck K. Trefoil factor family 3 expression in the oral cavity. Eur J Oral Sci 2009;117(6):636-643

18 Chaiyarit P, Jaresitthikunchai J, Phaonakrop N, Roytrakul S, Potempa B, Potempa J. Proteolytic effects of gingipains on trefoil factor family peptides. Clin Oral Investig 2018;22(2):1009-1018

19 Armitage GC. Development of a classification system for periodontal diseases and conditions. Ann Periodontol 1999;4(1):1-6

20 Khummuang S, Phanphrom W, Laopajon W, Kasinrerk W, Chaiyarit P, Pata S. Production of monoclonal antibodies against human trefoil factor 3 and development of a modified-sandwich ELISA for detection of trefoil factor 3 homodimer in saliva. Biol Proced Online 2017;19:14-1920

21 Romano F, Bongiovanni L, Bianco L, et al. Biomarker levels in gingival crevicular fluid of generalized aggressive periodontitis patients after non-surgical periodontal treatment. Clin Oral Investig 2018;22(2):1083-1092

22 Heitz-Mayfield LJA, Trombelli L, Heitz F, Needleman I, Moles D. A systematic review of the effect of surgical debridement vs non-surgical debridement for the treatment of chronic periodontitis. J Clin Periodontol 2002;29(Suppl 3):92-102, discussion $160-162$

23 Eberhard J, Jervøe-Storm PM, Needleman I, Worthington H, Jepsen S. Full-mouth treatment concepts for chronic periodontitis: a systematic review. J Clin Periodontol 2008;35(7):591-604

24 Nakamura-Kiyama M, Ono K, Masuda W, et al. Changes of salivary functions in experimental periodontitis model rats. Arch Oral Biol 2014;59(2):125-132

25 Nayar G, Gauna A, Chukkapalli S, Velsko I, Kesavalu L, Cha S. Polymicrobial infection alter inflammatory microRNA in rat salivary glands during periodontal disease. Anaerobe 2016;38:70-75

26 Önder C, Kurgan Ş, Altıngöz SM, et al. Impact of non-surgical periodontal therapy on saliva and serum levels of markers of oxidative stress. Clin Oral Investig 2017;21(6):1961-1969

27 Tabari ZA, Azadmehr A, Nohekhan A, Naddafpour N, Ghaedi FB. Salivary visfatin concentrations in patients with chronic periodontitis. J Periodontol 2014;85(8):1081-1085

28 Hendek MK, Erdemir EO, Kisa U, Ozcan G. Effect of initial periodontal therapy on oxidative stress markers in gingival crevicular fluid, saliva, and serum in smokers and non-smokers with chronic periodontitis. J Periodontol 2015;86(2):273-282

29 Novakovic N, Todorovic T, Rakic M, et al. Salivary antioxidants as periodontal biomarkers in evaluation of tissue status and treatment outcome. J Periodontal Res 2014;49(1):129-136

30 Bertl K, Schoiber A, Haririan H, et al. Non-surgical periodontal therapy influences salivary melatonin levels. Clin Oral Investig 2013;17(4):1219-1225

31 Türer ÇC, Durmuş D, Balli U, Güven B. Effect of non-surgical periodontal treatment on gingival crevicular fluid and serum endocan, vascular endothelial growth factor-A, and tumor necrosis factor-alpha levels. J Periodontol 2017;88(5):493-501

32 Peterson DE, Barker NP, Akhmadullina LI, et al. Phase II, randomized, double-blind, placebo-controlled study of recombinant human intestinal trefoil factor oral spray for prevention of oral mucositis in patients with colorectal cancer who are receiving fluorouracil-based chemotherapy. J Clin Oncol 2009;27(26):4333-4338 